\title{
Use of phytobiotics in animal husbandry and poultry
}

\author{
Vladimir Pashtetsky ${ }^{1}$, Pavel Ostapchuk ${ }^{1, *}$, Tatiana Kuevda ${ }^{1}$, Denis Zubochenko ${ }^{1}$, Sergey \\ Yemelianov $^{1}$, and Veronika Uppe ${ }^{1}$ \\ ${ }^{1}$ Crimean Research Institute of Agriculture, 150 Kievskaya St., Simferopol, Republic of Crimea, \\ 295493, Russia
}

\begin{abstract}
Scientists working in the field of animal nutrition have recently begun to pay more attention to biologically active substances contained in plants-phytobiotics. Their complex study (composition, effect on the body) is carried out only in the last 20-30 years. It was found that the use of plant components in animal husbandry (plant parts, essential oils, extracts) was accompanied by an increase in feed conversion, an overall improvement in production indicators, and an immunostimulatory effect.
\end{abstract}

\section{Introduction}

The search, characteristics, and application of natural antioxidants is the focus of numerous research groups worldwide. In light of discoveries made in the scientific field, the medicinal properties of plants are interesting because of their low toxicity, pharmacological activity and economic viability [1]. Natural antioxidants are now one of the most popular substances in food production and agriculture [2]. According to one theory, it is believed that the antioxidant properties of plants are developed in the course of evolution - based on photosynthetic activity. Therefore, each species, subspecies, variety of plants can be an object for assessing the antioxidant potential [1].

The ideal antioxidant should be easily absorbed and have to prevent free radical formation at physiologically significant levels. According to conducted animal studies, the antioxidant activity of extracts from summer savory (Satureia montana L.) in general, as well as its polyphenolic fraction, in particular, was shown [2].

In vitro addition of aqueous extract from savory twice increased the activity of catalase and superoxide dismutase (i.e enzymes that fight free radicals and wards off oxidative stress). The research results show that the mechanism of carvacrol and thymol action is in the destruction of the cytoplasmic membrane, which increases its permeability and depolarizes its potential [3].

\footnotetext{
${ }^{*}$ Corresponding author: ostapchuk_p@niishk.ru
} 


\section{Materials and methods}

Some recent studies report on antimicrobial properties of plant extracts containing polyphenols, which are particularly flavonoid-rich plant extracts. New antibiotics based on flavonoids are created and tested nowadays. Furthermore, the agents that boost the effect of other medicines due to the ability of flavonoids to suppress the mechanisms of multiple drug resistance (MDR), are also produced. Some natural compounds, including flavonoids can restore the effectiveness of medicines, thus overcoming the barrier of drug resistance [4].

The term "phytobiotics" includes a wide range of substances of biological origin, which can be divided, into four groups: 1) herbs, 2) parts of the plant (whole or treated, for example, roots, leaves, bark); 3) essential oils (hydrodistillated extracts of volatile plant compounds); and 4) oleoresins (extracts based on non-aqueous solvents) [4].

The effectiveness of phytobiotics is achieved thanks terpenoids (mono and sesquiterpenes, steroids), phenols (tannins), glycosides, alkaloids (present in the form of alcohols, alheids, ketones, esters, and lactones), flavonoids and glucosinolates.

In general, phytobiotics are herbal remedies with antibacterial effects. Phytobiotics contain primary and secondary plant compounds. Essential nutrients, i.e proteins, fats, and carbohydrates is considered as primary compounds while secondary compounds are described as EO, bitter substances, dyes, and phenolic compounds. Although the exact mechanisms of antimicrobial action of phytobiotics have yet not been clearly identified, some of the mechanisms possibly responsible for their beneficial properties, as defined by Vidanarachchi et al., Windisch and Kroismayr, include: 1) destruction of the cell membrane of pathogens; 2) modification of the cell surface affecting hydrophobicity and ,hence, their virulence; 3) stimulation of the immune system, in particular, activation of lymphocytes, macrophages and natural killer cells (NKC); 4) protection of the intestinal mucosa from colonization by bacterial pathogens [4].

Among phytobiotics, essential oils attract much attention due to their antimicrobial and growth-stimulating properties. Essential oils are compounds obtained from aromatic plants, herbs or spices by distillation or solvent extraction Many EO contain many active components. They are used to protect plants from damage caused by insects and bacteria. Each component may have a different mechanism of action, and these components may work synergistically. Thus, it can be concluded that the mechanism of action of EO is based on its chemical composition. Some example, in this regard is, the algorithm of action of thymol and carvacrol, which have similar antimicrobial effects but different mechanism of action due to differences in the location of the hydroxyl group.

The study of the properties of extracts and essential oils of plants is one of the most promising approaches to create preparations for diseases prevention and improving the productivity of poultry.

It is possible to strengthen the effect of biological influence of essential oils on an organism of an animal by combined application of EO and other additives based on vegetable raw materials $[4,12]$.

The productivity and health of birds in general, largely depend on the state of the digestive system. The intestinal mucous membrane of the farm animals and birds forms a large surface of contact with the external environment and represents the «main gate» for the penetration of antigens.

One of the most common infectious poultry diseases both worldwide and in the Russian Federation is salmonellosis. It is caused by bacteria of the Salmonella type. According to the Rosselkhoznadzor, salmonellosis is caused by the pathogenic bacterium Salmonella enteritidis. It is one of the most frequent infectious poultry diseases. This infection birds affects birds' gastrointestinal tract, lungs and joints causing huge economic losses at poultry 
farms. Salmonella can easily adapt to adverse environmental conditions, remain viable in dry and frozen foods, as well as exhibit high thermal stability. This microorganism is also often associated with outbreaks of foodborne diseases among humans.

Salmonella found in the digestive tract of humans with acute intestinal infections resulting from food poisoning are often resistant to most existing antibiotics: ampicillin, ceftriaxone, chloramphenicol, ciprofloxacin, phosphomycin, gentamicin, hygromycin, kanamycin, nalidixic acid, streptomycin, sulfamethoxazole, tetracycline, trimethoprimsulfamethoxazole, etc. [5].

Brenes and Roura proposed four mechanisms that describe the action of essential oils: sensory, metabolic, antioxidant, and antimicrobial. Moreover, Chowdhury et al., noted that the type and applied level of essential oil has a major impact on the productivity and availability of nutrients for birds. For example, $0.3 \mathrm{~g}$ of cinnamon oil per kilogram of diet can improve broiler chicken's growth performance and nutrient utilization. However, $0.6 \mathrm{~g} / \mathrm{kg}$ of clove oil and $0.4 \mathrm{~g} / \mathrm{kg}$ of ajwain oil has no effect on broilers.

\section{Results}

In order to develop the full genetic potential of poultry productivity, it is necessary to follow all parameters of the indoor environment and ensure a nutritious and balanced diet including biologically active feed additives.

Feed additive is a general name given to substances of different origin used to increase the viability and productivity of livestock and poultry. Antibiotics are used in most of the feed additives [5].

To improve the efficiency of fattening, antibiotics are added in feed in relatively small doses over a long period of time. Antibiotics, which are used in poultry feeding, stimulate growth, productivity, increase feed conversion, improve the incubation quality of eggs.

The use of antibiotics in poultry began in the 1940s. Nevertheless, there was not any initial confidence that they would actually be effective. Only in the mid-1940s, it became clear that their application improved the production performance of livestock farms. In this regard, the use of antibiotics has become widespread, but the mechanism of their action on the animal body remained unclear [6].

However, it would be wrong to say that the improvement in the performance of poultry farms at that time was achieved only thanks to the antibiotics. In tandem with them, there were also new advances in genetics, production management adjusted, feeding parameters improved, better - quality equipment, etc.

Moreover, experiments conducted in Denmark in the 1990s showed appeared that antibiotics withdrawal did not have the expected negative impact on poultry production. Several farms in the United States showed similar results. Scientists that worked there not only confirmed the previous Danish conclusions but also proved the downward production rates trend after antibiotics usage [6].

Shortly, after wards, turned out that the uncontrolled use of antibiotics negatively affects the body of animals and birds. For instance, some microorganisms can mutate and become resistant to antibiotics, and therefore result in reducing the positive effect of the medicinal product. Antibiotics also have some toxic and cumulative effect.

Currently, one of the most significant problems in modern industrial poultry are infectious diseases, the causative agents of which are Coccidia, Mycoplasma. They enter the body of birds with water, feed, after contact with rodents. These microorganisms cause outbreaks of infectious diseases. This is related to the industrial production where large number of animals are kept in small areas. This is one of the key factors of spreading various infections. The treatment of such diseases, cannot do without antibiotics. At the 
same time, the number of bacteria resistant to vancomycin, (the most effective antibiotic used to treat severe mixed human superinfections) continues to grow.

In 1994, in Denmark, the population consumed $24 \mathrm{~kg}$ of vancomycin, for therapeutic purposes. Though, animals ate 24 tons of avoparcin, belonging to the same group of antibiotics. They having similar mechanisms of action, so resistance to one of them is comparable to resistance to the other.

Over the past decade, molecular identification of bacterial pathogens has proved that antibiotic resistance can be transmitted between animals and humans. Feeding oxytetracycline to chickens led to tetracycline resistance of enterococci (E. coli) in chickens and the transfer of tetracycline resistance from chickens to service personnel [7].

In 1983, in East Germany, oxytetracycline was replaced by streptotricin (streptotricin antibiotic). This antibiotic was used throughout the country only in animal husbandry. In 1983, resistance to it was negligible. Two years later, resistant bacteria appeared in the intestines of pigs and in meat products.

In 1990, resistant to streptotricin bacteria were detected in the intestines of farmers, their families, citizens from municipal areas, and patients with urogenital infections. This resistance was probably because of meat products consumption. In 1987, resistance to other pathogens, including Shigella (a microorganism that is common only for humans) was also reported the context of emergence and spread of antibiotic resistance, enterococci have become an object of special interest.

Enterococci colonize the intestines of humans and animals, easily become resistance to antibiotics and transfer antibiotic - resistance genes [7].

In 1994, in many countries of the European Union, a large number of enterococci were repeatedly detected in water after poultry carcasses washing. However, by the end of 1997 , after banning of avoparcin, there was a $25 \%$ reduction in the number of bacteria resistant to it. At the same time there was also a decrease in the number of bacteria among from $12 \%$ in 1994 to $3.3 \%$ by the end of 1997 [8].

These studies clarify the role of the reservoir of antibiotic resistance in livestock and in humans.

According to the FAO (Food and Agriculture Organization) the EU spent 1.5 billion euros per year for the treatment of resistant pathogens in 2018; the cost of antibiotic resistance management in the USA was 0.1-10 billion dollars per year at the same time. By 2050 , antibiotic resistance will be able lead to a decline in world GDP by 2-3. $5 \%$ and damage of $\$ 100$ trillion.

According to World Health Organization (WHO), about 300 million people are expected to die due to antibiotic resistance in the next 35 years. Based on these global threats, in September 2017, the government of the Russian Federation signed decree No. 2045-R on the adoption of the "Strategy to prevent the spread of antimicrobial resistance in the Russian Federation for the period up to 2030".

The problems caused by the non-therapeutic use of antibiotic, or their consumption as part of feed additives can occur far beyond the country in which the animal or bird was raised because meat and meat products are traded around the world and bacterial populations spread very quickly crossing geographical boundaries.

\section{Discussion}

The inefficiency of existing drugs and treatments has led to the need to find new, effective means and ways to solve this problem. One such method is the use of essential oils have significant antiseptic, antibacterial, antiviral, antioxidant, antiparasitic, antifungal and insecticidal activity [9]. 
An important characteristic of essential oils and their components is hydrophobicity. It allows them to separate from lipids which are in the cell membrane of bacteria and mitochondria making them more permeable, disrupting cell structures. This eventually leads to bacterial cell death due to leakage of critical molecules and ions from the bacterial cell to a large extent [10].

Recent research Zabarna, I. et al. showed that essential oils slow down the formation flagella of E. coli. Deprived of flagella, these bacteria cannot move. This negatively affects their growth and reproduction.

Essential oils also have a pronounced probiotic effect, balancing the intestinal microflora. In addition to the ability to maintain the balance of intestinal microflora and immunomodulatory activity. Essential oils can stimulate the secretion of pancreatic enzymes in animals and birds.

An important quality of essential oils is the stability of their composition during use. Essential oils of oregano, cloves, cardamom, marjoram, laurel and fennel significantly changed during their storage [11].

The oxidation of some components of these essential oils was the main problem. The stability of labile components of fennel oil significantly increased in a mixture with essential oils of coriander and laurel. However, there is very little information about changes in the composition of oils during their storage $[12,6]$.

Another important reason for the use of essential oils is their coccidiostatic effect, which strengthens the effect of the medicinals used to eliminate this problem. Animals, in the feeds of which essential oils were used, showed the same reduction in the allocation of oocysts and the weakening of symptoms and lesions, as during the treatment with conventional coccidiostatics.

The right combination of organic acids with essential oils in poultry feeding, increases feed conversion, and activates pancreatic secretion [13].

Boskabady $\mathrm{M}$. et al conducted study to evaluate the effect of three different types of essential oils, namely cinnamon oil (CNO), clove oil (CLO), and ajvin oil (AJO) as an alternative to antibiotic growth-promoting (AGP) on growth productivity, nutrient utilization, nitrogen release, carcass characteristics, chemical and fatty acid composition of broiler chicken meat. Based on the results of this study, it can be concluded that CNO of $0.3 \mathrm{~g} / \mathrm{kg}$ can be used as an alternative to AGP in commercial broiler diets to improve growth characteristics and nutrient utilization while reducing nitrogen release to the environment [14].

In many sources it is reported about the positive effect of oils containing carvacrol on the motility of the gastrointestinal tract. There is an increase in the secretion of digestive enzymes, there and normalization of the intestinal microbiota.

The results of studies conducted by Allaoua M. et al. showed that the mechanism of action of carvacrol and thymol is the destruction of the cytoplasmic membrane, which increases its permeability and depolarizes its potential. Similarly, carvacrol acts with respect to a variety of targets in bacterial membranes and cytoplasm. Plant $\mathrm{J}$ et al. in vitro studies show that carvacrol inhibits biofilm formation [15].

An experiment was conducted to assess the effect of carvacrol supplements in four concentrations $(0,120,200$ and $300 \mathrm{mg} / \mathrm{kg}$ diet $)$ on the amount of Escherichia coli, Campylobacter spp in the cecum at all levels of supplementation. Thus, an alternative to antibiotic use demonstrated as Awaad M. H. H et al. noted that carvacrol consumption linearly reduces feed intake, feed conversion rate, and body weight gain at all levels of the supplement addition.

Addition of $240 \mathrm{mg} / \mathrm{kg}$ of the preparation, that contains $25 \%$ of thymol and $25 \%$ of carvacrol as active components, to broiler chickens, improved intestinal histomorphology. Decreased inflammatory response and increased specific immune response against $\mathrm{C}$. 
perfringens-challenged. Feeding of $300 \mathrm{mg} / \mathrm{kg}$ not only improved the histomorphological properties of the small intestine (height and area of the villi), but also reduced the amount of E. coli as a pathogen microorganism in the chicken intestine [16].

Zeng Z. et al. studied the effect of feed additives with thymol on immunity of broiler chickens. This component had a stimulating effect on the immune response [17-18]. For example, Ane Orchard et al. investigated the effect of thymol on the smooth muscles of the trachea and ileum and the ciliary movement of the respiratory system in rats. They found that thymol has a dose-dependent antispasmodic property and increases mucosal transport due to the movement of cilia [19].

In addition, the antispasmodic effect of thyme extract, which is presumably associated with phenolic compounds of volatile oils such as thymol, was demonstrated. The relaxant effect of carvacrol on the smooth muscle of the trachea of guinea pigs was higher than that of theophylline [20].

The combination of a low dose of essential oil containing thymol $(50 \mathrm{mg} / \mathrm{kg})$ and $1 \mathrm{~g} / \mathrm{kg}$ of Na-butyrate reduced the content of Salmonella in the cecum. Recently, the food industry has become increasingly interested in natural compounds, either for direct use or for joint use with other compounds [21].

In vivo studies, when essential oils were used alone or in combination, showed a clear inhibition of Clostridium perfringens and E. coli in the intestine, the integrity of the intestinal wall was preserved. During the experiment, the weight gains of group of birds consuming Satureja khuzistanica essential oil was higher compared to the birds of the control group [22].

One of the well-known mechanisms of antibacterial activity is related to their hydrophobicity, which disrupts cell membrane permeability and cell homeostasis due to loss of cell components, influx of other substances, or even cell death. Gram-negative bacteria are more resistant to the action of essential oil than gram-positive ones because of their hydrophilic components in the outer membrane.

Interactions between antimicrobials can lead to three different effects, i.e. synergistic, additive, or antagonistic. Synergy is achieved by combining two antimicrobial compounds with antibacterial activity exceeding the sum of the antibacterial activity of the individual components. The additive effect is achieved by combining antimicrobials producing an antimicrobial effect equal to the sum of the individual compounds. The antagonistic effect leads to a decrease in the antimicrobial activity of the two compounds in combination compared to their individual antimicrobial activity.

The antimicrobial activity of the essential oil can only depend on the main components that make up the whole oil. When tested in binary or triple combinations, various synergistic antimicrobial actions have been reported.

For instance, García-García et al. found that carvacrol and thymol were the most synergistic binary combination against L. innocua. Futhermore, carvacrol, thymol, and eugenol were the most active in the triple combination [23].

The components of essential oils, namely thymol and carvacrol, show synergism with penicillin in relation to E. coli and S. typhimurium. Carvacrol has a synergistic effect in combination with ampicillin and nitrofurantoin against Klebsiella oxytoca, which was isolated from animal feed, while thymol in this case had no effect [24].

In a study conducted by Rosato et al. it has been reported that oregano oil in combination with gentamicin showed synergism against B. cereus, B. subtilis and one strain of $\mathrm{S}$. aureus [25].

Zataria multiflora essential oil (Shiraz oregano) showed synergistic activity with vancomycin against methicillin-sensitive Staphylococcus aureus (MSSA) and 12 clinical MRSA isolates, although FIC data for individual strains were not specified. In studies conducted by Hood et al. combinations of Australian tea tree volatile oil (Melaleuca 
alternifolia) with aminoglycoside antibiotics have been investigated. Yersinia enterocolitica, Serratia. marcescens and one strain of S. aureus were among the bacterial species found to have synergism with gentamicin. It was found that the FIC index is on the border between additivity and synergism against Acinetobacter baumannii, B. subtilis and another strain of S. aureus. In addition, tea tree oil along with tobramycin has synergism against E. coli and S. aureus [25].

Aminoglycosides have been found to inhibit protein synthesis and tea tree oil damages the bacterial cytoplasmic membrane; this may have been an example of multi-purpose synergy. Ampicillin and gentamicin, together with clove oil, have been tested for synergism against a number of periodontic pathogens. FIC index of less than 0.5 were found for ampicillin against Streptococcus mutans, S. sobrinus and Streptococcus gordonii, for gentamicin against Streptococcus sanguinis, S. criteci and Porphyromonas gingivalis.

In an in vitro study conducted by Duarte et al. the combination of coriander essential oil with gentamicin, chloramphenicol, ciprofloxacin and tetracycline against Acinetobacter baumannii showed efficacy, which was therefore an indicator of possible synergistic interaction against two reference strains of Acinetobacter baumannii (LMG 1025 and LMG 1041) with FIC index equal to 0.047 and 0.37 respectively. Study conducted by Micciche A. et al. showed that this interaction in vitro can improve the antimicrobial efficacy of tetracycline, ciprofloxacin and gentamicin and may contribute to the re-sensitization of Acinetobacter baumannii to the action of chloramphenicol [24-25].

In most cases, the study of the combination of essential oils of Eucalyptus camaldulensis with conventional antibiotics (gentamicin, ciprofloxacin and polymyxin B) showed a synergistic antibacterial effect even in some re-sensitized strains of A. baumannii with multiple drug resistance (MDR). The synergistic effect was obtained by combining the essential oil with antibiotics, for example, aminoglycosides, tetracyclines, fluoroquinolone, which can serve as the basis for the development of a new therapy against drug-resistant bacteria [26-30].

The effect of using essential oils contained in the products of coniferous plants processing in poultry farming is noted: cross chickens gives a significant increase in live weight $(3.5-7.5 \%)$ during the growth period in our research [27] (fig. 1).

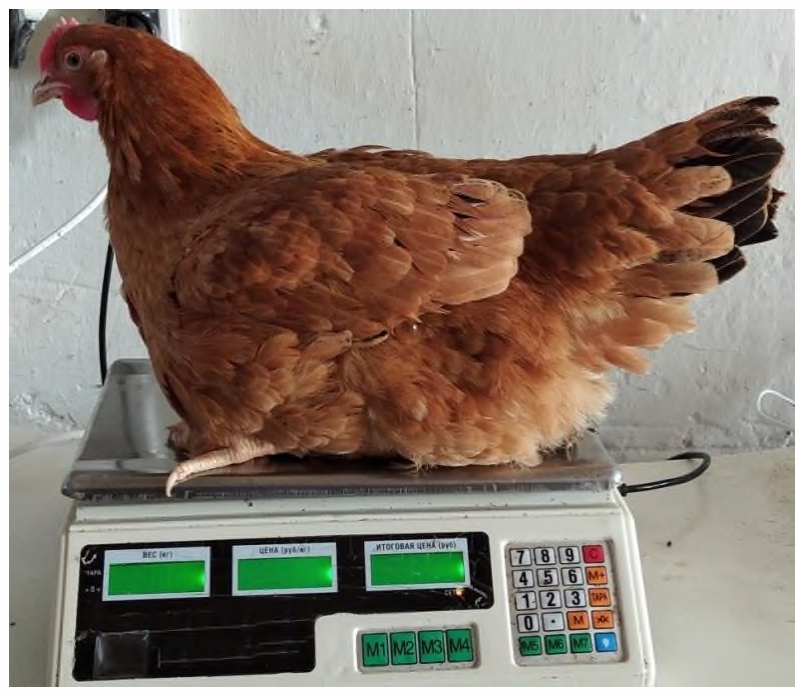

Fig. 1. Chicken of the experimental group.

Essential oils, sprayed through the air, in veterinary medicine can be a promising alternative to disinfectants. Essential oils Mentha piperita, Origanum vulgare, Citrus 
bergamia, Salvia officinalis and Syzygium aromaticum have been shown to be an effective alternative for disinfection of hatching eggs (effective dose is $0.045 \mathrm{ml} / \mathrm{m} 3$ ). The use of essential oils is safe for the health of service personnel [28-32].

\section{Conclusion}

In conclusion, it can be stated that plants are an unlimited renewable source of biologically active substances, including up to 12 thousand compounds, of which no more than 2 thousand are currently described.

The prevailing opinion about the harmlessness of medicinal plants does not quite correspond to reality. A significant disadvantage of herbal medicine is a relatively slow therapeutic effect and unstable chemical composition, which does not allow accurately calculating the necessary dose. Essential oils have an extremely complex chemical composition. This property of oils provides a wide range of effects on the living organism, and at the same time the difficulties of standardization, because the chemical composition of oils depends on the region of growth, weather conditions, time of harvesting, ets.

\section{References}

1. T. Candan, A. Bağdatlı, CBU J. of Sci. 13(2), 279-291 (2017) DOI 10.18466/cbayarfbe. 319752

2. M. Marzoni, R. Chiarini, A. Castillo, I. Romboli, M. De Marco, Anim. Sci. Pap. Rep. (32)4, 359-368

URL: http://freejournal.umm.ac.id/files/file/Effects\%20of\%20dietary\%20natural\%20antioxid ant $\% 20$ supplementation $\% 20$ on\%20broiler\%20chicken $\% 20$ and $\% 20$ Muscovy\%20duck $\% 20$ meat $\% 20$ quality.pdf

3. M. Alagawany, M. Ezzat Abd El-Hack, A Reviews on Trends and Advances in Safeguarding Terrestrial Aquatic Animal Health and Production 3(2), 73-79 (2015) DOI: http://dx.doi.org/10.14737/journal.aavs/2015/3.2s.73.84

4. M. Gheisar, I.H. Kim, Italian Journal of Animal Science (17), 1-6 (2017) http://dx.doi.org/10.1080/1828051X.2017.1350120

5. S. Diaz-Sanchez, et al., Botanical alternatives to antibiotics for use in organic poultry production Poultry Science, 94, 1419-1430 (2015)

6. K. Karásková, P. Suchý, E. Straková, Czech Journal of Animal Science, 12, 521-530 (2015) DOI: 10.17221/8594-CJAS

7. C. Agyare, V. Boamah, C. Zumbi, F. Osei, Antimicrobial Resistance: A Global Threat, 33 - 42 (2018) http://dx.doi.org/10.5772/intechopen.79371

8. M. Gopi, et al., A Feed additive in poultry nutrition, 2, 1-7 (2014)

9. K. Gopal, A. Narang, Journal of Advanced Veterinary and Animal Research, 1, 156 162 (2014) DOI: 10.5455/javar2014.a36

10. J. Plant, B. Stephens, Medicinal \& Aromatic Plants, 4, 2-5 (2015) http://dx.doi.org/10.4172/2167-0412.1000185

11. I. Zabarna, O. Yakubchak, T. Taran, I. Jacenko, S. Midyk, Research Journal of Pharmaceutical, Biological and Chemical Sciences, 10(3), 391-397 (2019)

12. Y. Tarakhovsky, Y. Kim, B. Abdrasilov, E. Muzafarov, Flavonoids: biochemistry, biophysics, medicine (Pushchino, Online Journal, 310, 2013) 
13. A. Cerisuelo, F. Marín, E. Gómez, J. M. de la Fuente, R. Durán, C. Fernández, Poultry Science, 93, 599-606 (2014) http://dx.doi.org/ 10.3382 P-599-606

14. Boskabady, M. Alitaneh, A. Alavinezhad, BioMed Research International, 1-11 (2014) http://dx.doi.org/10.1155/2014/569087

15. M. Allaoua, et al. Journal of Applied Microbiology, 1162-1171 (2017)

16. Guyard-Nicod'eme, M. Keita, S. Quesne, M. Amelot, T. Poezevara, B. Le Berre, J. S'anchez, P. Vesseur, A. Mart'1n, Poultry Science, 298-305 (2016) http://dx.doi.org/10.3382/ps/pev303 P -298-304.

17. E. Zeng, Journal of Animal Science and Biotechnology, 6, 1-10 (2015) DOI: 10.1186/s40104-015-0004-5

18. A. Saki, J. Salary, Iran Poultry Science Journal, 3(2), 165-170 (2015)

19. A. Orchardand, S. Vuuren, Complementary and Alternative Medicine, 20-92 (2017) https://doi.org/10.1155/2017/4517971.

20. M. Awaad, M. Elmenawey, A. Kawkab, Veterinary World, 7(3), 284-290 (2014)

21. O. İlhak, O. İncili, H. Durmuşoğlu, Annals of Animal Science, (17)1, 271-280 (2017) DOI: 10.1515/aoas-2016-0047

22. H. Khosravinia, Journal of Applied Animal Research, (44)1, 273-280 (2016) http://dx.doi.org/10.1080/09712119.2015.1031781

23. S. Chouhan, K. Sharma, S. Guleria, Medicines, 4, 1-21 (2017) DOI: $10.3390 /$ medicines 4030058

24. A. Micciche, J. Michael, J. Rothrock, Y. Yang, Frontiers in Microbiology, 10, 279-291 (2019) DOI: 10.3389/fmicb.2019.01058

25. M. Adaszyńska, D. Szczerbińska, Annals of Animal Science, 17(2), 317-335 (2017) DOI: 10.1515/aoas-2016-0046

26. M. Mousa, A. Osman, H. Abdel Hady, Journal of veterinary medicine and animal health, 10, 255-263 (2017)

27. V. Pashtetskiy, P. Ostapchuk, O. Postnikova, V. Korotkiy, T. Kuevda, A. Pashtetskaya, IOP Conference Series: Earth and Environmental Science, 422, 012071 (2020) DOI: 10.1088/1755-1315/422/1/012071

28. S. Movahhedkhah, B. Rasouli, A. Seidavi, D. Mazzei, V. Laudadio, Microflora Animals, 9, 87-93 (2019) DOI:10.3390/ani9030087

29. R. Castillo-López, E. Gutiérrez-Grijalva, N. Leyva-López, L. López-Martínez, J. Heredia, The Journal of Animal \& Plant Sciences, 349-359 (2017) ISSN: 1018-7081

30. S. Akyildiz, M. Denli, Animal Science, LIX, 71 - 74 (2016)

31. A. Alavinezhad, M. Boscabady, Phytoterapy Research, 28, 1739 - 1748 (2014) DOI: 10.1002/ptr.5200

32. I. Piskaeva, V. Dolganyuk, S. Noskova, O. Chaplygin, Foods and Raw Materials, (5)2, 137-144 (2017) DOI: 10.21179/2308-4057-2017-2-137-144 